\title{
PAIRS OF MATRICES, ONE OF WHICH COMMUTES WITH THEIR COMMUTATOR*
}

\author{
GERALD BOURGEOIS ${ }^{\dagger}$
}

\begin{abstract}
Let $A, B$ be $n \times n$ complex matrices such that $C=A B-B A$ and $A$ commute. For $n=2$, we prove that $A, B$ are simultaneously triangularizable. For $n \geq 3$, we give an example of matrices $A, B$ such that the pair $(A, B)$ does not have property L of Motzkin-Taussky, and such that $B$ and $C$ are not simultaneously triangularizable. Finally, we estimate the complexity of the Alp'in-Koreshkov's algorithm that checks whether two matrices are simultaneously triangularizable. Practically, one cannot test a pair of numerical matrices of dimension greater than five.
\end{abstract}

Key words. Nilpotent matrix, Property L, Commutator, Quasi-commute.

AMS subject classifications. 15A27, 15A22.

\section{Introduction.}

Definition 1.1. i) We say that the $n \times n$ complex matrices $A, B$ quasi-commute if both $A$ and $B$ commute with $A B-B A$.

ii) The $n \times n$ complex matrices $A, B$ are said to be simultaneously triangularizable $(S T)$ if there exists an invertible matrix $P$ such that $P^{-1} A P$ and $P^{-1} B P$ are upper triangular.

Consider the following standard result.

TheOREm 1.2. (Little McCoy's Theorem [6]) If A and B quasi-commute, then they are $S T$.

In this article, we deal with pairs of $n \times n$ complex matrices $(A, B)$ such that only $A$ commutes with $A B-B A$. If $(A, B)$ is such a pair, then for any complex numbers $\lambda, \mu,\left(A+\lambda I_{n}, B+\mu I_{n}\right)$ is another one. Then we may assume that $A$ and $B$ are invertible. In the sequel, we put $C=A B-B A$. We introduce notation and definitions that will be used in the paper.

Notation. i) If $U$ is a square matrix, then $\sigma(U)$ and $\chi_{U}$ denote the spectrum and the characteristic polynomial of $U$.

ii) Denote by $I_{n}$ and $0_{n}$ the identity matrix and the zero matrix of dimension $n$.

${ }^{*}$ Received by the editors on May 20, 2011. Accepted for publication on June 4, 2011. Handling Editor: Roger A. Horn.

${ }^{\dagger}$ GAATI, Université de la Polynésie française, BP 6570, 98702 FAA'A, Tahiti, Polynésie Française (gerald.bourgeois@upf.pf). 
Definition 1.3. (See [7]) A pair $(A, B)$ of complex $n \times n$ matrices is said to have property $L$ if for a special ordering of the eigenvalues $\left(\lambda_{i}\right)_{i \leq n},\left(\mu_{i}\right)_{i \leq n}$ of $A, B$, the eigenvalues of $x A+y B$ are $\left(x \lambda_{i}+y \mu_{i}\right)_{i \leq n}$ for all values of the complex numbers $x, y$.

REMARK 1.4. (See [7]) If $A, B$ are $S T$, then the pair $(A, B)$ has property L but, except if $n=2$, the converse is false.

Several known results are gathered in the following Proposition.

Proposition 1.5. Let $A, B$ be complex $n \times n$ matrices. We assume that $C$ and $A$ commute. Then $C$ is nilpotent and the pair $(B, C)$ has property L. Moreover, if $A, B$ are invertible, then $A^{-1} B^{-1} C, B^{-1} A^{-1} C$ and $B^{-1} C$ are nilpotent.

Proof. By Jacobson's Lemma, see [5, Lemma 2], $C$ is nilpotent. According to [3], one has, for every $t \in \mathbb{R}$ and for any $A, B \in \mathcal{M}_{n}(\mathbb{C}), e^{t A} B e^{-t A}=B+t C+\frac{t^{2}}{2 !}[A, C]+$ $\frac{t^{3}}{3 !}[A,[A, C]]+\cdots$. By an analytic continuation, this equality works also for complex numbers $t$. Here, we obtain for every $t \in \mathbb{C}$

$$
e^{t A} B e^{-t A}=B+t C
$$

and therefore, $\sigma(B+t C)=\sigma(B)$. It follows that the pair $(B, C)$ has property L. Now we assume that $A, B$ are invertible. We have

$$
A^{-1} C B^{-1}=C A^{-1} B^{-1}=A B A^{-1} B^{-1}-I_{n} .
$$

By $\left[9\right.$, Theorem 2], $A B A^{-1} B^{-1}-I_{n}$ is nilpotent. Since

$$
\sigma\left(A^{-1} B^{-1} C\right)=\sigma\left(C A^{-1} B^{-1}\right)=\{0\} \text { and } \sigma\left(B^{-1} A^{-1} C\right)=\sigma\left(A^{-1} C B^{-1}\right)=\{0\},
$$

we conclude that $A^{-1} B^{-1} C$ and $B^{-1} A^{-1} C$ are also nilpotent. By [9, proof of Theorem 1], we obtain that $C B^{-1}$ is nilpotent (or equivalently $B^{-1} C$ is nilpotent).

2. Positive and negative results. We may wonder whether $A$ and $B$ are $S T$ or, at least, the pair $(A, B)$ has property L. We have a positive answer in the following case.

Definition 2.1. A complex matrix $A$ is said to be non-derogatory if for every $\lambda \in \sigma(A)$, the number of Jordan blocks of $A$ associated with $\lambda$ is 1 .

Proposition 2.2. If $A$ is a non-derogatory matrix and if $A C=C A$, then $A$ and $B$ are $S T$.

Proof. Necessarily, $C$ is a polynomial in $A$. According to [2, Theorem 1], $A$ and $B$ are $S T$. 
REMARK 2.3. i) Note that the set of derogatory matrices is included in the set $N S$ of non-separable matrices, that is they have at least one multiple eigenvalue. The set $N S$ is an algebraic variety in $\mathcal{M}_{n}(\mathbb{C})$ of codimension 1 . Therefore, it is a null set in the sense of Lebesgue measure (see [8] for an outline of the proof).

ii) If we fix the matrix $A$, then the equation $A(A B-B A)=(A B-B A) A$ is linear in the unknown $B$. More precisely $B \in \operatorname{ker}(\phi)$ where $\phi: X \rightarrow A^{2} X+X A^{2}-2 A X A$. Hence,

$$
\phi=A^{2} \otimes I+I \otimes\left(A^{T}\right)^{2}-2 A \otimes A^{T}=\psi^{2},
$$

where $\psi=A \otimes I_{n}-I_{n} \otimes A^{T}$. Thus, if $\sigma(A)=\left(\lambda_{i}\right)_{i}$, then $\sigma(\psi)=\left(\lambda_{i}-\lambda_{j}\right)_{i, j}$ and $\sigma(\phi)=\left(\left(\lambda_{i}-\lambda_{j}\right)^{2}\right)_{i, j}$. The quantity

$$
i(A)=\frac{\operatorname{dim}\left(\operatorname{ker}\left(\psi^{2}\right)\right)-\operatorname{dim}(\operatorname{ker}(\psi))}{n^{2}}
$$

indicates the existence of a matrix $B$ such that $A B-B A$ and $A$ commute and such that $A, B$ are not $S T$.

Now we prove our main result.

Proposition 2.4. i) If $n=2$ and $A C=C A$, then $A$ and $B$ are $S T$.

ii) If $n \geq 3$, then there exists a pair $(A, B)$ such that

- $A C=C A$,

- $(A, B)$ does not have property $L$,

- $B$ and $C$ are not $S T$.

Proof. i) According to Proposition 2.2, we may assume that $A$ is derogatory, that is, $A$ is a scalar matrix, which gives the conclusion immediately.

ii) It is sufficient to find such a counterexample $\left(A_{0}, B_{0}\right)$ when $n=3$. Indeed, if $n>3$, consider the pair $\left(A_{0} \bigoplus 0_{n-3}, B_{0} \bigoplus 0_{n-3}\right)$.

Now suppose that $n=3$ and that $A_{0}$ is the derogatory matrix $A_{0}=\left[\begin{array}{lll}0 & 1 & 0 \\ 0 & 0 & 0 \\ 0 & 0 & 0\end{array}\right]$. Then $\psi$ is nilpotent and we have the equalities

$$
\operatorname{dim}(\operatorname{ker}(\psi))=5, \operatorname{dim}\left(\operatorname{ker}\left(\psi^{2}\right)\right)=8 \text { and } i\left(A_{0}\right)=\frac{1}{3} .
$$

The associated matrices $B$ are the matrices with a zero entry in position $(2,1)$. We choose

$$
B=\left[\begin{array}{lll}
0 & 0 & 0 \\
0 & 0 & 1 \\
1 & 0 & 0
\end{array}\right]
$$


- $\left(A_{0}, B\right)$ does not have property L because $\sigma\left(A_{0}\right)=\{0\}$ and for every pair of complex numbers $(t, x), \chi_{t A_{0}+B}(x)=x^{3}-t$.

- We observe that $\operatorname{Tr}\left(B^{2} C^{2}\right)=-1$. This implies that $B$ and $C$ are not $S T$.

REMARK 2.5. We can prove i) by reducing $A$ to Jordan canonical form and examining the cases in which $A$ is diagonalizable or not.

Proposition 2.6. For every $n \geq 4$, there exists a derogatory matrix $A_{1}$ such that $A_{1}$ and each associated matrix $B$ are $S T$.

Proof. We take $n=4$ and $A_{1}=\left[\begin{array}{cc}0_{2} & I_{2} \\ 0_{2} & 0_{2}\end{array}\right]$. Note that $A_{1}$ is in Weyr canonical form (see [10]) and not in Jordan canonical form. One has

$$
\operatorname{dim}(\operatorname{ker}(\psi))=8, \operatorname{dim}\left(\operatorname{ker}\left(\psi^{2}\right)\right)=12 \text { and } i\left(A_{1}\right)=\frac{1}{4}<i\left(A_{0}\right)
$$

The associated matrices $B$ are in the form $B=\left[\begin{array}{cc}E & F \\ 0 & G\end{array}\right]$ where $E, F, G$ are arbitrary $2 \times 2$ complex matrices. Let $U, V$ be $2 \times 2$ invertible complex matrices such that $U^{-1} E U$ and $V^{-1} G V$ are upper triangular. We remark that $P^{-1} A_{1} P$ and $P^{-1} B P$ are upper triangular where $P=\operatorname{diag}(U, V)$.

3. How to determine whether two matrices are $S T$. In general, how can one determine whether two $n \times n$ complex matrices are $S T$ or not? McCoy's Theorem (see Section 2.4 of [4]) is an available tool, but it does not give a finite verification procedure.

The following theorem leads to an algorithm to check whether two matrices are ST.

Theorem 3.1. (Alp'in-Koreshkov, see [1, Theorem 6]) Two $n \times n$ complex matrices $A$ and $B$ are $S T$ if and only if for every $k \in\left[\left[1, n^{2}-1\right]\right]$, each matrix of the form $U_{1} \cdots U_{k}(A B-B A)$ ( where, for every $i, U_{i}$ is $A$ or $B$ ) has a zero trace.

REMARK 3.2. If the entries of $A, B$ are in a subring $K$ of $\mathbb{C}$, then all computations are performed in $K$.

Using Theorem 3.1, we must check that $2^{n^{2}}-2$ matrices have a zero trace. If $A, B$ are not $S T$, then the test stops when it finds a matrix with non-zero trace. If $A, B$ are $S T$, then the test requires $2^{n^{2}}$ matricial multiplications in $\mathcal{M}_{n}(\mathbb{C})$. We can deduce the following.

Proposition 3.3. The complexity of the computation induced by Theorem 3.1 is equivalent to $2^{n^{2}} n^{3}$ complex multiplications. 
Experiments. We used a cluster provided with 16 GB of RAM.

For the following $4 \times 4$ matrices, that are $S T$,

$$
\begin{aligned}
A & =\left[\begin{array}{cccc}
308831848 & 3514720569 & -2393248600 & -933664618 \\
1653458482 & -2646203334 & 1951033145 & 1428485078 \\
185766230 & -909262575 & 2221156990 & 78496990 \\
1349546744 & -2237843658 & 4279424410 & 96552841
\end{array}\right], \\
B & =\left[\begin{array}{cccc}
-277500618 & 34522275 & 180434913 & -933966414 \\
2348943678 & 1523928630 & -700130673 & 1316048154 \\
-97303050 & -203818485 & 577843890 & 179268180 \\
394577946 & 431913075 & -336185991 & 967683108
\end{array}\right],
\end{aligned}
$$

the duration of the test was less than one second and the used memory was about 90 MB.

In dimension five, there is a big storage at the end of the penultimate step. Precisely, at this stage, we store $2^{23}$ matrices of dimension 5 . We considered a pair of numerical $5 \times 5$ matrices, that were $S T$ and such that their entries were integers with absolute value at most 1000. Then the duration of the test was 2 minutes 26 seconds.

In dimension six, the maximal storage theoretically uses tens of terabytes of RAM and consequently this test only works to show eventually that two matrices are not $S T$.

Acknowledgment. The author thanks professor R. Horn for bringing this problem to his attention, and D. Adam for many valuable discussions.

\section{REFERENCES}

[1] Y. Alp'in and N. Koreshkov. On the simultaneous triangulability of matrices. Math. Notes, 68:552-555, 2000.

[2] G. Bourgeois. How to solve the matrix equation $X A-A X=f(X)$. Linear Algebra Appl., 434:657-668, 2011.

[3] J.E. Campbell. On a law of combination of operators bearing on the theory of continuous transformation groups. Proc. London Math. Soc., 28:381-390, 1897.

[4] R.A. Horn and C.R. Johnson. Matrix Analysis. Cambridge University Press, Cambridge, 1985.

[5] N. Jacobson. Rational methods in the theory of Lie algebras. Ann. of Math., 36:875-881, 1935.

[6] N. McCoy. On quasi-commutative matrices. Trans. Amer. Math. Soc., 36:327-340, 1934.

[7] T.S. Motzkin and O. Taussky. Pairs of matrices with property L. II . Trans. Amer. Math. Soc., 80:387-401, 1955.

[8] P. Neumann and C. Praeger. Cyclic matrices over finite fields. J. London. Math. Soc. (2), 52:263-284, 1995.

[9] C.R. Putnam and A. Wintner. On the spectra of group commutators. Proc. Amer. Math. Soc., 9:360-362, 1958.

[10] H. Shapiro. The Weyr characteristic. Amer. Math. Monthly, 196:919-929, 1999. 